\title{
ANÁLISE FOLIAR EM CINCO ESPÉCIES DE EUCALIPTOS *
}

\author{
H. P. HAAG ** \\ J. R. SARRUGE ** \\ G. S. Oliveira ** \\ F. POGgiani \\ C. A. FERREIRA $* * * *$
}

RESUMO

Foram coletadas 200 folhas recem maduras de meia altura $(\mathrm{H}=11 \mathrm{~m})$ da copa das espécies $E$. graandis, E. microcorys, $E$. saligna de sete anos de idade, localizadas em solo latossolo, na região de Mogi Guaçu e Areia Branca, SP. As folhas foram analisadas para $\mathrm{N}, \mathrm{P}, \mathrm{K}, \mathrm{Ca}, \mathrm{Mg}, \mathrm{S}, \mathrm{B}, \mathrm{Cu}, \mathrm{Fe}, \mathrm{Mn}$ e $\mathrm{Zn}$ com os objetivos de detectar exigências nutricionais distintas para as espécies, assim como correlacionar o teor dos nutrientes com o volume cilindrico de madeira $\left(\mathrm{m}^{3} / \mathrm{ha}\right)$. Constatou-se diferenças significativas na composição química entre as espécies. A espécie $E$. gnandis apresenta um teor mais elevado em $\mathrm{N}, \mathrm{P}, \mathrm{K}$ e S. As concentrações mais baixas dos nutrientes são encontradas no $E$. microcorys. Foram constatadas diferenças significativas entre os locais nas espécies com exceção de $\mathrm{Mg}, \mathrm{B}, \mathrm{Cu}, \mathrm{Fe}$ e $\mathrm{Zn}$. Teores elevados em Mn foram constatados nas folhas de todas as espécies. $O$ eucalípto de maior produção é o E. grandis, sendo o E. microcorys o de menor produção. Foi constatada correlação positiva entre $\mathrm{N}, \mathrm{P}, \mathrm{K}, \mathrm{S}$ e Fe nas folhas e produção cilindrica de madeira.

\section{INTRODUÇÃO}

Nos últimos vinte anos o uso de fertilizantes como prática cultural no manejo de florestas tem se expandido consideravelmente, mormente nos países industrializados. O uso de fertilizantes tem incentivado a pesquisa na área de nutrição, particularmente a análise de tecido para fins de diagnóstico de avaliação do estado nutricional (Gessel et alii,

* Entregue para publicação em 20-01-1977.

* Departamento de Quamica, E.S.A. "Luiz de Queiroz", USP, S.P.

*** Departamento de Silvicultura, E.S.A "Luiz de Queiroz", USP, S.P.

**** Champiur Papel e Celulose S/A, S.P. 
1960; Leyton, 1959; International Potasa Institute, 1967; Tamm, 1964; Mustanoja \& Leaf, 1965; Leaf, 1973; Malavolta et alii, 1974).

O eucalípto constitue uma espécie florestal de grande importância para o Brasil. O emprego da análise foliar nesta essencia, acha-se, ainđa, nos seus primórdios, destacando-se contudo os trabalhos de Mello et alii (1960); Melo et alii (1961), Haag (1965) e Mello et alii (1970).

O presente trabalho deve por objetivos:

1 - Detectar e avaliar, através da análise foliar, diferenças na concentração de nutrientes nas espécies.

2 - Correlacionar os teores dos nutrientes com o volume de madeira produzido.

\section{MATERIAL E MÉTODOS}

Foram coletadas, em abril de 1976, 200 folhas recém maduras de cada árvore (quatro repetições), provenientes de ramos situados a meia altura $(\mathrm{H}=11 \mathrm{~m})$ das copas, dirigidas aos quatro pontos cardiais. $\mathrm{O}$ material coletado foi tratado e analisado para $\mathrm{N}, \mathrm{P}, \mathrm{K}, \mathrm{Ca}, \mathrm{Mg}, \mathrm{S}, \mathrm{B}, \mathrm{Cu}$, $\mathrm{Fe}, \mathrm{Mn}$ e $\mathrm{Zn}$ de acordo com as instruções contidas em Sarruge \& Haag (1974).

As folhas coletadas provieram de espécies de eucalíptos localizados em um solo latossol vermelho-amarelo, barro arenoso (Areia Branca, Lat. $22^{\circ} 20^{\prime}$; Long. $47^{\circ} 008$ ) e de um solo latossol vermelho-amarelo, barro arenoso (Mogi Guaçu, Lat. 22 $52^{\prime}$; Long. $47^{\circ} 00^{\prime}$ ). A altitude de ambos locais é de aproximadamente $580 \mathrm{~m}$. Os dois povoamentos foram instalados em junho de 1969 , no espaçamento de $3,00 \times 2,50 \mathrm{~m}$, recebendo na ocasião $200 \mathrm{~g} /$ planta de nutrientes da fórmula 5-10-1 por cova.

As folhas coletadas pertenciam as seguintes espécies de eucalíptos: Eucalyptus saligna Sm.; Eucalyptus resinifera Sm., Eucalyptus microcorys F. Muell; Eucalyptus robusta Sm.; Eucalyptus grandis W. Hill ex Maiden. Na região a precipitação média anual foi de $1.300 \mathrm{~mm}$ e a temperatura média anual de $20,3^{\circ} \mathrm{C}$. Segundo a classificação climática de Koppen a região situa-se no tipo Cwa.

RESULTADOS E DISCUSSÃO

Solos - Caracterização analítica

Local - Horto Areia Branca 
Geologia - sedimentos argilo-arenosos retrabalhados

Relevo - normal, suave ondulado, longo

Vegetação - eucalípto

Drenagem - boa

Perfil - n. ${ }^{\circ} \mathrm{T}_{5}-\mathrm{AB}$, Registro 3546-3550

Fonte: G. Ranzani, Equipe Levantamento CES ( $\left.{ }^{*}\right)$

Resultados Analíticos - Tabela 1

Local - Horto Mogi-Guaçu

Geologia - sedimentos argilo-arenosos

Vegetação - eucaliptos

Drenagem - boa

Perfil n. ${ }^{\circ} \mathrm{T}_{i-}$-MG; Registro $3568-3577$

Fonte - G. Ranzani e Equipe Levantamento CES (*)

Resultados analíticos - Tabela 2

Os solos são classificados em Latossolo Vermelho-Amarelo - barro arenoso (Areia Branca) e Latossolo Vermelho-Amarelo - barro argiloso arenoso (Mogi-Guaçu). São solos de fertilidade natural muito baixa. Baseando-se na interpretação dos dados analíticos em Catani \& Jacintho (1974) estes solos apresentam baixos níveis de $\mathrm{P}$ solúvel, teores baixos em $\mathrm{K}, \mathrm{Ca}, \mathrm{Mg}$ trocáveis e acidez elevada (Tabelas 1 e 2). O teor de prótons extraídos é baixo. Estes solos possuem baixa atividade de argila (CTC) e porcentagem de saturação em bases, muito baixa. A diferença mais marcante, sob o ponto de vista analítico entre os solos é a concentração de Al trocável. O solo da região de Areia Branca apresenta um nível médio, sendo alta na região de Mogi-Guaçu. Digno de nota o fato da produção de madeira $\left(\mathrm{m}^{3} / \mathrm{ha}\right)$ foi superior em Mogi-Guaçu, apesar do nível alto de Al. (Tabela 2) As plantas tolerantes ao Al são poucas e pouco conhecidas. Pode se supor que todas as plantas que crescem nos Latossolos com altos teores de Al sejam tolerantes. Chenery (1948) classifica a família das Myrtaceae como sendo pouco acumuladores de Al.

(*) Departamento de Solos e Geologia, E.S.A. "Luiz de Queiroz", USP., Piracicaba, SP. 


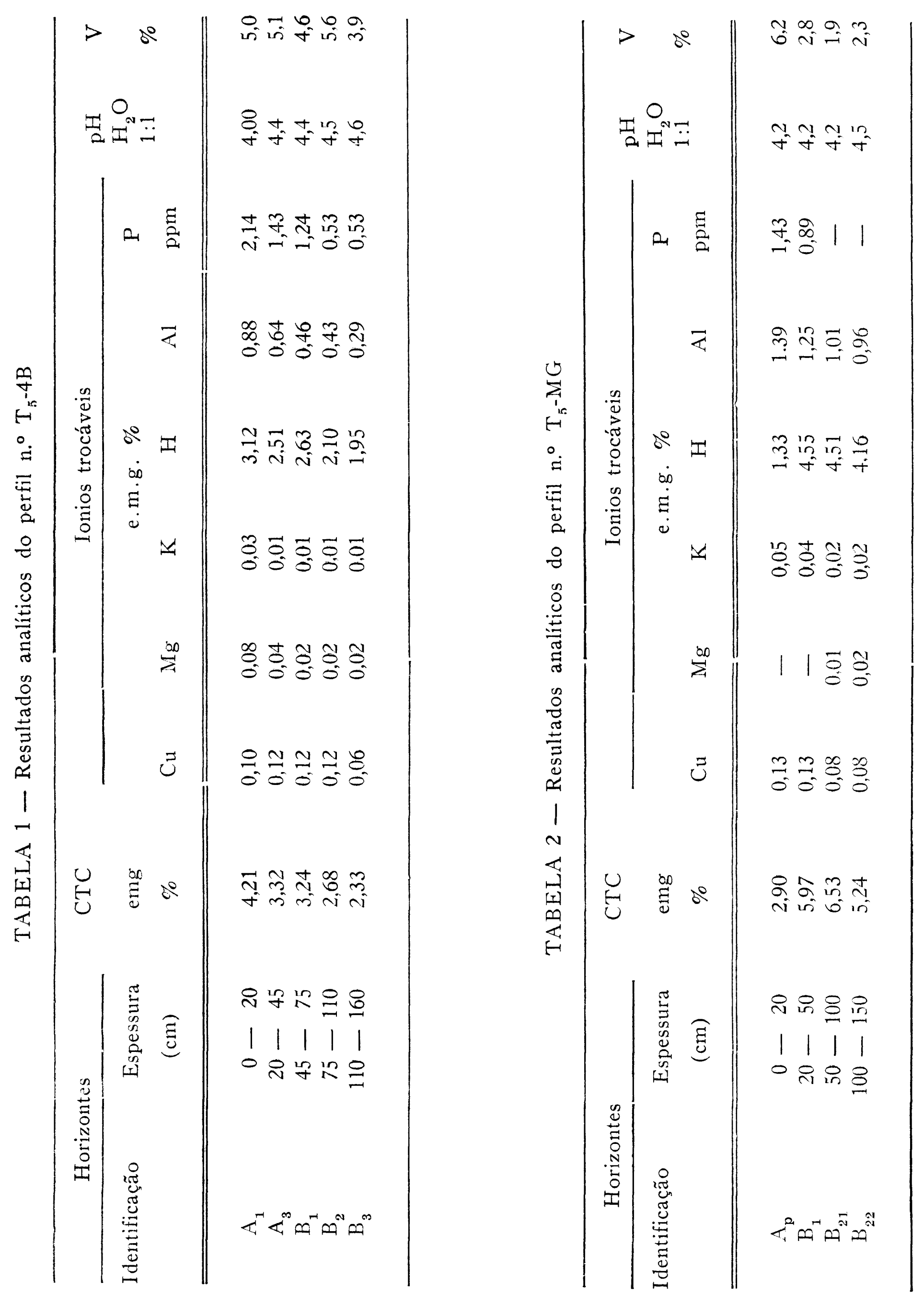




\section{Concentração de Macronutrientes}

A concentração porcentual dos macronutrientes, na matéria seca, nas folhas recém maduras, acha-se assinalada na Tabela 3. Observa-se, uma diferença na concentração de $\mathrm{N}$ nas espécies em função do solo e no mesmo solo entre as espécies. A espécie $E$. grandis mostra uma concentração mais elevada deste nutriente em ambos os solos.

Haag et alii (1961), determinaram o teor de $\mathrm{N}$ nas folhas de $E$. alba e $E$. grandis com dois anos de idade, oriundas de um solo de cerrado, sem assinalarem sintomas de carência e encontraram valores de $2,07 \%$ e $2,22 \%$, respectivamente. Os valores encontrados no presente trabalho são inferiores e sugerem uma desnutrição nas espécies de eucalíptos.

As concentrações de $\mathrm{P}$ encontradas são extremamente baixas e indicam uma carência neste elemento, quando confrontadas com os dados de Mello et alii (1960), citados em Malavolta et alii (1974). O E. grandis apresenta uma maior capacidade de extração de $\mathrm{P}$, traduzida por uma maior concentração destes elementos nas folhas.

Mello et alii (1970) em um ensaio fatorial $3 \times 3 \times 3 \times 2$ para $N, P, K$ e calcáreo na região de Mogi-Guaçu, empregando E. saligna com cinco anos de idade obtiveram uma resposta significativa do $\mathrm{P}$ sobre a produção de madeira. A base econômica de $P$ para época foi de $172 \mathrm{~kg} / \mathrm{ha}$ de $\mathrm{P}_{2} \mathrm{O}_{5}$.

Folhas coletadas de E. camalduleuses e E. gomphocephala com doze anos de idade ap:esentaram segundo Metro \& Beaucorps (1968), valores oscilando em torno de $0,60 \%$ a $0,62 \%$ de $\mathrm{K}$ na matéria seca. Em condições brasileiras Haag et alii (1961) apresentaram teores de $1,04 \%$ a $1,14 \%$ de $\mathrm{K}$ nas folhas de $E$. alba e $E$. grandis com dois anos de idade. Os dados do trabalho indicam uma insuficiência de $\mathrm{K}$ em todas as espécies, em ambos os solos. Por ocasião da coleta das folhas, observou-se sintomas nas folhas de $\boldsymbol{E}$. microcorys, na região de Areia Branca, que assemelhavam a deficiência de $\mathrm{K}$-necrose nas margens das folhas e pequenas áreas necrobadas espalhadas no limbo.

As concentrações de Ca diferem pouco entre as espécies, destacando-se contudo a concentração mais elevada no E. robusta. Os valores encontrados foram baixos em confronto com os da literatura. Assim, Metro \& Beaucorps (1958), encontraram para E. cameldulensis e E. gomphocephala, valores de $1,07 \%$ e $1,50 \%$ de Ca respectivamente. No Brasil, Haag et alii (1961) apontam valores de $1,28 \%$ e $1,15 \%$ de $\mathrm{Ca}$ em $E$. alba e E. grandis. Apesar das baixas concentrações em Ca não houve correlação com o volume cilíndrico de madeira produzido, como será visto adiante. 


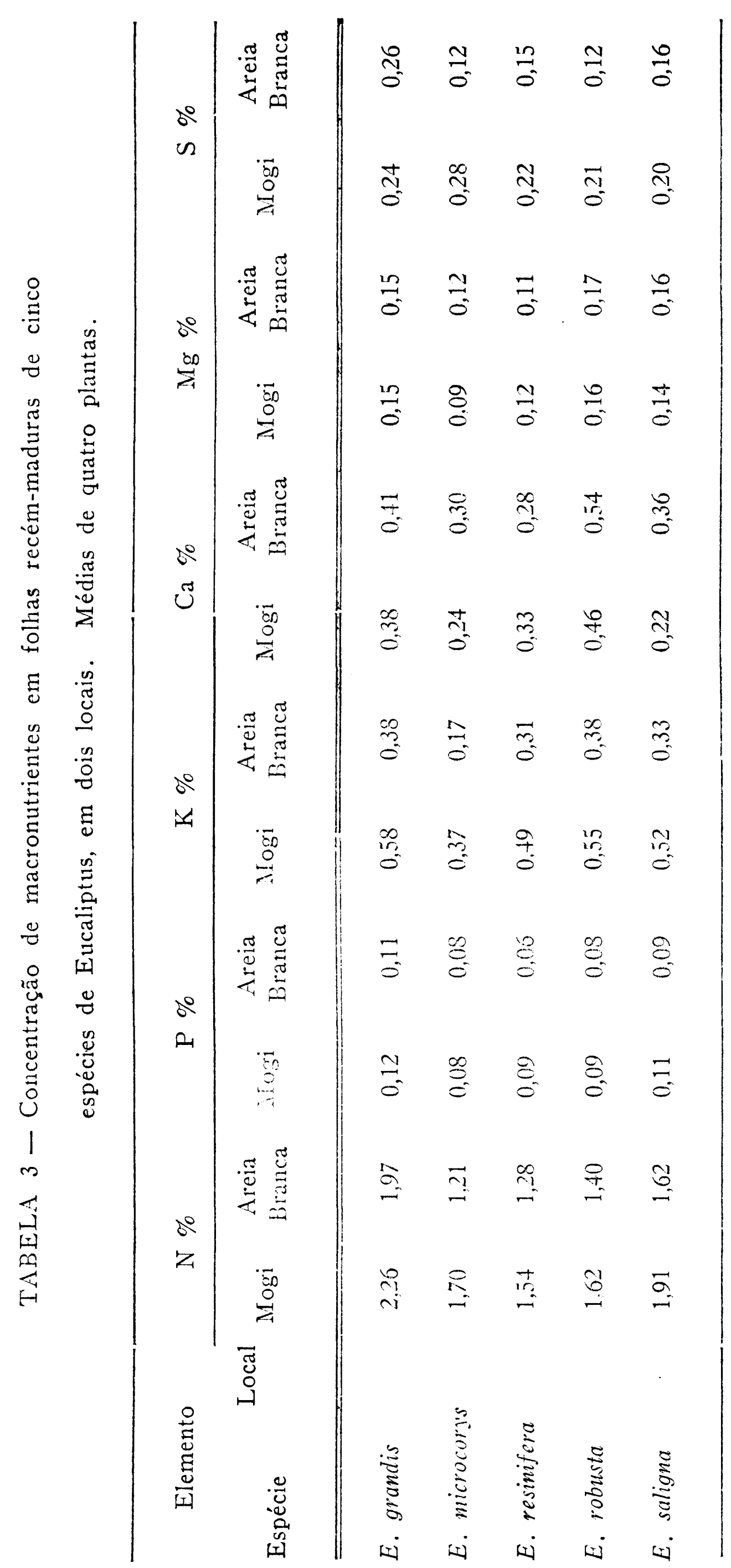


Apesar das baixas concentrações de $\mathrm{Mg}$ em todas as espécies, não foram identificado sintomas de carência. Mello et alii (1961), cultivaram $E$. alba, em areia lavada omitindo o $\mathrm{Mg}$, obtiveram um quadro sintomatológico quando a concentração de $\mathrm{Mg}$ atingiu o nível de $0,21 \%$ na matéria seca.

Os teores de $\mathrm{S}$ encontrados, acham-se dentro dos limites citados na literatura. Um dos poucos trabalhos em que o $\mathbf{S}$ é citado pertence a Mello et alii (1961). Os autores cultivaram E. alba em areia lavada com e sem adição de $\mathrm{S}$ e encontraram os valores de $0,26 \%$ e $0,16 \%$ de $\mathrm{S}$ nas folhas, com e sem adição de $\mathrm{S}$ ao substrato. Chama atenção o fato de que a concentração de $\mathbf{S}$ é mais elevada nas espécies cultivadas na região de Mogi-Guaçu do que em Areia Branca. Uma possível explicação, talvez, seja a maior proximidade da Usina Champion Papel e Celulose S.A., em relação ao Horto de Mogi-Guaçu (cerca de $3,5 \mathrm{~km}$, em linha reta).

Diferenças na concentração dos macronutrientes nas espécies de eucalíptos foram detectadas através da análise de efeito de espécies nos dois povoamentos e os resultados acham-se expressos na Tabela 4. Destaca-se o $E$. grandis por seus teores mais elevados em $\mathrm{N}, \mathrm{P}, \mathrm{K}, \mathrm{Mg}$ e $\mathbf{S}$.

TABELA 4 - Concentração média de macronutrientes em folhas recém maduras de cinco espécies de Eucalyptus de Mogi Guaçu e Areia Branca. Média de oito plantas. Letraas não comuns expressam diferenças significativas pelo teste de Tukey a $5 \%$

\begin{tabular}{lcccccc}
\hline $\begin{array}{c}\text { Elementos } \\
\text { Espécies }\end{array}$ & $\mathrm{N} \%$ & $\mathrm{P} \%$ & $\mathrm{~K} \%$ & $\mathrm{Ca} \%$ & $\mathrm{Mg} \%$ & $\mathrm{~S} \%$ \\
\hline E. grandis & $2,12 \mathrm{a}$ & $0,12 \mathrm{a}$ & $0,48 \mathrm{a}$ & $0,39 \mathrm{~b}$ & $0,15 \mathrm{ab}$ & $0,25 \mathrm{a}$ \\
E. microcorys & $1,46 \mathrm{c}$ & $0,08 \mathrm{c}$ & $0,27 \mathrm{~b}$ & $0,27 \mathrm{c}$ & $0,11 \mathrm{c}$ & $0,20 \mathrm{~b}$ \\
E. resinifera & $1,41 \mathrm{c}$ & $0,08 \mathrm{c}$ & $0,40 \mathrm{a}$ & $0,30 \mathrm{bc}$ & $0,12 \mathrm{bc}$ & $0,19 \mathrm{bc}$ \\
E. robusta & $1,51 \mathrm{bc}$ & $0,08 \mathrm{c}$ & $0,46 \mathrm{a}$ & $0,50 \mathrm{a}$ & $0,17 \mathrm{a}$ & $0,16 \mathrm{a}$ \\
F & $1,77 \mathrm{~b}$ & $0,10 \mathrm{~b}$ & $0,43 \mathrm{a}$ & $0,29 \mathrm{bc}$ & $0,15 \mathrm{ab}$ & $0,18 \mathrm{bc}$ \\
& $19,75^{* *}$ & $25,00^{* *}$ & $9,15 * *$ & $12,55 * *$ & $6,50^{* *}$ & $9,22^{* *}$ \\
\hline D.M.S. (Tukey & 0,27 & 0,01 & 0,11 & 0,11 & 0,04 & 0,04 \\
& $11,4 \%$ & $11,2 \%$ & $18,7 \%$ & $21,3 \%$ & $20,4 \%$ & $15,4 \%$ \\
C.V. a 5\%) & & & & & &
\end{tabular}


Esta maior demanda de nutrientes refletiu-se na produção de madeira, conforme pode se ver ver na Tabela 5 .

TABELA 5 - Concoentraação média de macronutrientes em folhas recém maduras de cinco espécies de Eucalyptus. Média de vinte plantas. Lctras não comuns expressam diferenças significativas

\begin{tabular}{cccc}
\hline Llementoc & Mogi & $\begin{array}{c}\text { Areia } \\
\text { Branca }\end{array}$ & F \\
\hline $\mathrm{N} \%$ & $1,81 \mathrm{a}$ & $1,50 \mathrm{~b}$ & $26,70 * * *$ \\
$\mathrm{P} \%$ & $0,10 \mathrm{a}$ & $0,08 \mathrm{~b}$ & $27,00 * *$ \\
$\mathrm{~K} \%$ & $0,50 \mathrm{a}$ & $0,31 \mathrm{~b}$ & $61,26 * *$ \\
$\mathrm{Ca} \%$ & 0,32 & 0,38 & 5,30 \\
$\mathrm{Mg} \%$ & $0,13 \mathrm{a}$ & $0,14 \mathrm{a}$ & $1,12 \mathrm{~ns}$ \\
$\mathrm{~S} \%$ & $0,23 \mathrm{a}$ & $0,16 \mathrm{~b}$ & $50,67 * *$ \\
\hline
\end{tabular}

O E. microcorys apresenta os teores mais baixos em N, P, K, Ca e $\mathrm{Mg}$, sendo os menos produtivos em volume cilíndrico de madeira. Chama igualmente atenção, o baixo teor de $\mathrm{K}$ e $\mathrm{Mg}$ nas folhas, atestando ser uma espécie que tem preferência para solos mais férteis. O $E$. robusta e $E$. resinifera apresentam concentrações dos macronutrientes ligeiramente superior ao $E$. microcorys, mas inferiores ao $E$. grandis e E. saligna.

A análise do efeito do local nas espécies por elementos nos dois tipos de solos acha-se exposta na Tabela 5. Observa-se um aumento significativo em todos os nutrientes, com exceção do $\mathrm{Ca}$ e $\mathrm{Mg}$, nos eucalíptos cultivados na região de Mogi-Guaçu. Chama atenção o teor elevado de $\mathbf{S}$ na região de Mogi-Guaçu, pelos motivos já assinalados.

\section{Concentração de Micronutrientes}

A concentração dos micronutrientes nas folhas, expressa em ppm na matéria seca é apontada na Tabela 6 . Torna-se muito difícil diagnosticar se estes níveis podem ser considerados baixos, médios ou altos, por falta de padrões comparativos. Trabalho conduzido por Haag (1965), cultivando $E$. tereticornis em solução nutritiva purificada, obteve os seguintes valores para plantas deficientes: B - $67 \mathrm{ppm}$; $\mathrm{Cu}$ 9,3 ppm; Fe - 1,86 ppm; Mn - 5,3 ppm; Zn - 5,0 ppm. 


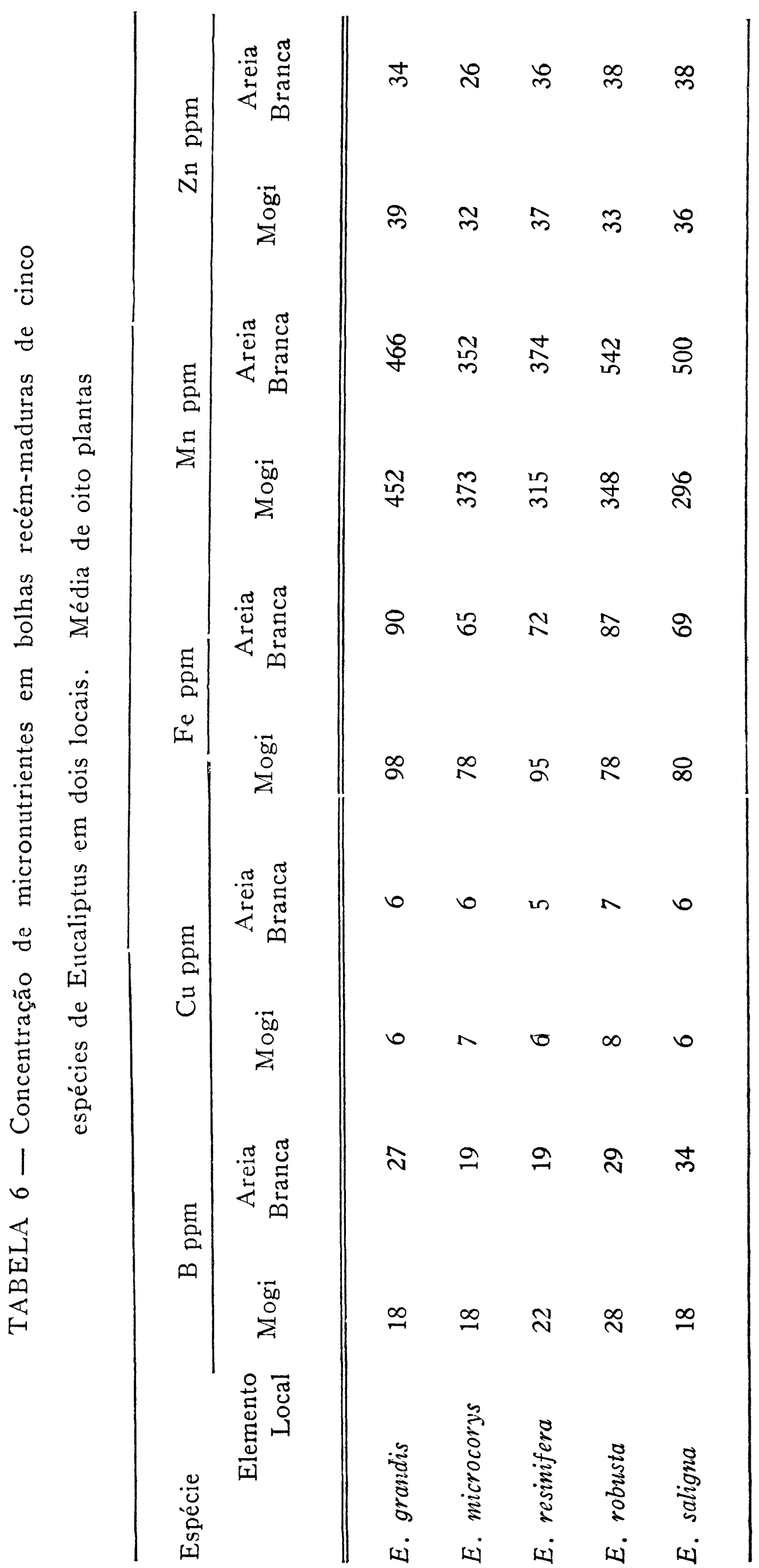


E interessante assinalar os altos valores encontrados para Mn, nas folhas dos eucalíptos. Níveis altos de Mn nas plantas estão associado a solos de acidez elevada, como os detectados no presente trabalho.

O efeito das espécies na concentração dos micronutrientes, acha-se assinalado na Tabela 7 . Somente a concentração de $\mathrm{Cu}$ acusou diferenças entre as espécies $E$. resinifera e $E$. robusta, de pouco significado biológico.

A análise do efeito de local na concentração dos micronutrientes nas folhas das espécies acha-se assinalada na Tabela 8, observa-se uma

QUNDRO 7 - Concentração média de micronutrientes em folhas recém-maduras de cinco espécies de eucaliptus de Mogi Guaçu e Areia Branca. Média de oito plantas. Letras não comuns expressam diferenças significativas pelo teste de Tukey a $5 \%$

\begin{tabular}{|c|c|c|c|c|c|}
\hline $\begin{array}{l}\text { Elementos } \\
\text { Esprécies }\end{array}$ & $\mathrm{B}$ ppm & $\mathrm{Cu}$ ppm & Fe ppin & Mn ppm & $\mathrm{Zn} \mathrm{ppm}$ \\
\hline E. arandis & 23 & $6 \mathrm{ab}$ & 94 & 459 & 35 \\
\hline E. microcorys & 19 & 6 ab & 72 & 363 & 29 \\
\hline 1. resinifera & 21 & $5 \mathrm{~b}$ & 83 & 344 & 29 \\
\hline E. robusta & 28 & $7 a$ & 82 & 445 & 36 \\
\hline E. saligna & 26 & $6 \mathrm{ab}$ & 74 & 398 & 37 \\
\hline $\mathrm{F}$ & $2,21 \mathrm{~ns}$ & $4,81 * *$ & $1,81 \mathrm{~ns}$ & $2,33 \mathrm{~ns}$ & $0,58 \mathrm{~ns}$ \\
\hline D.M.S. (Tukey a $5 \%$ ) & - & 1,4 & - & - & - \\
\hline C.V. & $31,7 \%$ & $15,3 \%$ & $22,2 \%$ & $23,1 \%$ & $34,9 \%$ \\
\hline
\end{tabular}

TABBELA 8 - Concentração média de mieronutrientes em folhas recém-maduras de cinco espécies de Eucalyptus. Média de vinte plantas. Letras não comuns expressão diferenças significativas

\begin{tabular}{lccc}
\hline B ppm & Local & Areia & F \\
\hline \hline Cu ppm & 21 & 26 & 4,07 \\
Elemento & 6 & 6 & 2,27 \\
Fe ppm & 86 & 76 & 2,72 \\
Mn ppm & 357 a & $447 \mathrm{~b}$ & $9,44 * *$ \\
Zn ppm & 35 & 34 & 0,04 \\
\hline
\end{tabular}


diferença significativa, no teor de $\mathrm{Mn}$, sendo que, os eucaliptos da região de Areia Branca apresentam teor mais elevado deste micronutriente, o que reflete as condições de acidez elevada nos solos.

\section{Volume de madeira produzido}

A Tabela 9 expressa a produção de madeira em $\mathrm{m}^{3}$ / ha e estereos/ /ha/ano das cinco espécies estudadas. Observa-se inicialmente uma diferença significativa na produção de madeira nos dois tipos de solos; sendo que as plantas da região de Mogi-Guaçu apresentam uma produção muito mais elevada, independente da espécie. O E. grandis supera nitidamente as demais espécies quando cultivado em solo de fertilidade um pouco mais elevada de Mogi-Guaçu. Entre as espécies, a menos produtiva é o $E$. microcorys em ambos os solos, produtividade baixa ligada a ausência de solos de maior fertilidade. As espécies $E$. resinifera, $E$. robusta e $E$. saligna, não diferem entre si. A produção média do Estado de São Paulo, para $E$. saligna segundo Mello et alii (1970) situa-se em torno de 28 a 30 estéreos/ha/ano aos sete anos de idade. Pelos dados do presente trabalho observa-se que na região de Mogi-Guaçu todas as espécies atingiram estes limites, apesar da adubação diminuta empregada por ocasião do plantio.

TABELA 9 - Volume cilindrico $\left(\mathrm{m}^{3} / \mathrm{ha}\right)$ e estereos/ha/ano de cinco espécies de Eucalyptus. Média de quatro repetições. Medições tomadas em $1 / 5 / 76$. Letras não comuns expressam diferenças significativas pelo teste de Tukey a $5 \%$

\begin{tabular}{|c|c|c|c|c|c|}
\hline Espécie & Local & $\mathrm{m}^{3} / \mathrm{ha}$ & $\begin{array}{c}\text { Mogi Guaçu } \\
\text { cstéreos } \\
\text { ha/ahos }\end{array}$ & $\begin{array}{l}\text { Areia } 1 \\
\mathrm{~m}^{3} / \mathrm{ha}\end{array}$ & $\begin{array}{l}\text { estéreos } \\
\text { ha/ahos }\end{array}$ \\
\hline E. grandis & & 581,0875 a & 66,40 & $197,1013 \mathrm{c} \mathrm{d}$ & 22,52 \\
\hline E. microcorys & & $274,2468 c$ & 31,11 & 89,7088 e & 10,24 \\
\hline E. resinifera & & $375,7425 \mathrm{~b}$ & 42,92 & $171,3400 \mathrm{~d}$ & 19,58 \\
\hline E. robusta & & $397,3700 \mathrm{~b}$ & 45,41 & $143,4470 \mathrm{~d}$ & 16,39 \\
\hline E. saligna & & $374,5930 \mathrm{~b}$ & 42,80 & $130,7430 \mathrm{~d}$ & 14,94 \\
\hline
\end{tabular}

\begin{tabular}{|c|c|c|}
\hline & Espécies & $26,04 * *$ \\
\hline$F=$ & Locais & $379,15 * *$ \\
\hline & Locais $x$ Espécies $\quad \ldots \ldots \ldots \ldots \ldots \ldots \ldots$ & $7,13 *$ \\
\hline & $\begin{array}{l}\text { C.V. }=15,09 \% \\
\text { (Tukey) D.M.S. } 5 \%=99,4658\end{array}$ & \\
\hline
\end{tabular}


Complementando o presente trabalho foram calculadas as equações de regressão e índice de correlação entre produção de madeira (y) e teor dos nutrientes (x) nas folhas das cinco espécies de eucaliptos.

As regressões representativas do teor do elemento e a produção de madeira, são as seguintes:

$$
\begin{aligned}
& \mathrm{N}-=261,45 \mathrm{x}-158,57 \quad \mathrm{r}=0,60 * * \\
& \mathrm{P}^{\mathrm{y}}=4.476,80 \mathrm{x}-126,24 \quad \mathrm{r}=0,60 * * \\
& \mathrm{~K}^{\mathrm{y}}=715,27 \mathrm{x}-1,76 \quad \mathrm{r}=0,58 * * \\
& \mathrm{~S}-\mathrm{y}^{\mathrm{y}}=1.431,74 \mathrm{x}+2,13 \quad \mathrm{r}=0,51 * * \\
& \underset{\mathrm{y}}{\mathrm{Fe}-\mathrm{y}}=2,7732 \mathrm{x}+48,63 \quad \mathrm{r}=0,34 * *
\end{aligned}
$$

Para os demais nutrientes não há correlação significativa entre produção de madeira e teor de nutriente nas folhas.

Estes resultados apontam a necessidade em se conduzir ensaios de adubação, empregando-se níveis crescentes dos elementos.

\section{CONCLUSÕES}

As espécies de eucaliptos apresentam concentração diferente dos macronutrientes nas folhas.

Concentração mais elevada de $\mathrm{N}, \mathrm{P}, \mathrm{K}$ e $\mathrm{S}$ são encontradas no $\boldsymbol{E}$. grandis. As concentrações mais baixas dos macronutrientes com exceção do S são encontradas no $E$. microcorys.

Os eucaliptos da região de Mogi-Guaçu apresentam concentrações mais elevadas nos macronutrientes $\mathrm{N}, \mathrm{P}, \mathrm{K}$ e $\mathrm{S}$.

Os eucaliptos não diferem na concentração dos macronutrientes nas folhas.

As espécies mais produtivas em ambos os solos em volume cilíndrico de madeira é o $E$. grandis.

A espécie menos produtiva é o $E$. macrocorys.

Os eucaliptos na região de Mogi-Guaçu são mais produtivos do que os da região de Areia Branca. 
Há correlação positiva entre produção de madeiras e os nutrientes N, P, K, S e Fe.

\section{AGRADECIMENTOS}

Aos Srs. Antonio Aparecido de Camargo e Agenor Rabello dos Santos, esporistas-colhedores de sementes do IPEF pelas possibilidades de coleta do material.

\section{A Champion Papel e Celulose S.A., São Paulo, pela autorização da coleta de folhas e informações prestadas.}

\section{SUMMARY}

\section{FOLIAGE ANALYSIS OF FIVE EUCALYPTUS SPECIES}

Two hundred recent mature leaves were collected, at april 1976, from each of the upper crown part of to years old plants (E. grandis, E. microcorys, E. resinifera, E.robusta, E. saligna), established on two Red Yellow Latossol site at Mogi Guaçu and Areia Branca, State of São Paulo, Brasil. Chemical analysis were run for $\mathrm{N}, \mathrm{P}, \mathrm{K}, \mathrm{Ca}, \mathrm{Mg}, \mathrm{S}, \mathrm{B}, \mathrm{Cu}, \mathrm{Fe}$, $\mathrm{Mn}$ and $\mathrm{Zn}$ in order to detect nutritional differences between the species. The highest concentration of $\mathrm{N}, \mathrm{P}, \mathrm{K}$ and $\mathrm{S}$ were founded in E. grandis; while E. microcorys exhibited the lowest concentrations in nutrients. High elevels in $\mathrm{Mn}$ were observed in all species due the acid soils. The yields in $\left(\mathrm{m}^{3} / \mathrm{ha}\right.$.) of wood have varied considerably within the species. The higher production of wood was of the species $E$. grandis and the lower was due $E$. microcorys. A positive correlation were observed between nutrient concentration $(x)$ and yield of wood (y) for the elements and is expressed by the following equations:

$$
\begin{array}{rl}
\mathrm{N}_{\mathrm{y}}=261.45 \mathrm{x}-158.51 & \mathrm{r}=0.60 * * \\
\mathrm{P}_{\mathrm{y}}=4.476 .80 \mathrm{x}-126.24 & \mathrm{r}=0.60 * * \\
\mathrm{~K}_{\mathrm{y}}=715.27 \mathrm{x}-1.76 & \mathrm{r}=0.58^{* *} \\
\mathrm{~S}_{\mathrm{y}}=1.431 .74 \mathrm{x}-2.13 & \mathrm{r}=0.51 * * \\
\mathrm{Fe}_{\mathrm{y}}=2.773 .2 \mathrm{x}+48.63 & \mathrm{r}=0.34 *
\end{array}
$$

\section{LITERATURA CONSULTADA}

CATANI, R.A. e A.O. JACINTHO, 1974. Análise química para Avaliar a fertilidade do solo. Boletim Técnico Científico n. ${ }^{\circ} 37$. E.S.A. "Luiz de Queiroz", Piracicaba, Brasil, 57 p.

CHENERY, E.M. 1948. Aluminum in plants and its relation to plant pigments. Ann. Bot. 12: 121-136. 
GESSEL, S.P.; K.Y. TURNBULL; F.T. TREMBLAY, 1960. How to fertilize trees and measure response. Nat. Plant Fodl Inst. Wash, D.C. 67 p.

HAAG. H.P.; H.A. MELlO; F.A.F. DE MELLO; M.O.C. BRASIL SOB. ${ }^{\circ}$; A.A. VEIGA, 1961. Composição de Eucalyptus alba Reinw e Eucalyptus grandis (Mill) Marden. Resultados preliminares. 2. ${ }^{a}$ Conferência Mundial de Eucalipto 2: 1329-1334, São Paulo, Brasil.

HAAC, H.P., 1965. Dados não publicados.

INTERNATIONAL POTASH INSTITUTE, 1967. Collogguium on forest fertilization. Proc. 5th Colloquium. Int. Potash Inst., Berne, Switzerland, $379 \mathrm{p}$.

LEAF, A.L., 1973. Plant Analysis as an Aid in Fertilizing Forests. Em "Soil Textury and Plant Analysis". Soil Sci. Soc. of Amer., Madison, Wis 491 p.

LEYTON, L., 1958. The mineral requirements of forest plants. Encyl. Plant Physiol. 4: 1026-1029.

MALAVOLTA, E.; H.P. HAAG; F.A.F. DE MELLO; M.O.C. BRASIL SOB., 1974. Nutrição Mineral e Adubação de Plantas Cultivadas. Livraria Pioneira Editora, Sĩo Paulo, 727 p.

MELLO, H.A.; A.A. VEIGA; F.A.F. DE MELLO; M.O.C. BRASIL SOB. ${ }^{\circ}$; H.P. HAAG; E. MALAVOLTA, 1960. Composição de Pinus elliottiin, Engelman. Fertilité, 9: $11-14$.

MELlO, F.A.F. de; H.P. HAAG; M.O.C. BRASIL SOB. ${ }^{\circ}$ W.E. ACCORSI; E. MALAVOLTA; S. ARZOLA, 1961. Efeitos da adubação nitrogenada, fosfatada e potássica sobre o "Stand" de eucalipto. 2. a Conferência Mundial do Eucalipto 2: 933-838. São Paulo, Brasil.

MELlO, H.A.; J. MASCARENHAS SOB. ${ }^{\circ}$; J.W. SIMÕES; H.T.Z. COUTO, 1970. Resultados da aplicação de fertilizantes minerais na produção de madeira de Eucalypius saligna Sm. em solos de cerrado do Estado de São Paulo. IPEF 10: 7-26.

METRO, A.E.; G. DE BEAUCORPS, 1958. Influência dos povoamentos de eucaliptos sobre a evolução dos solos arenosos ao Rharb. Fertilité, 4: 3-13.

MUSTANOJA, K.J.; A.L. LEAF, 1965. Forest fertilization research. Bot. Rev. 31: 151-246.

SARRUGE, J.R.; H.P. HAAG, 1974. Analises Qumicas em Plantas. E.S.A. "Luiz de Queiroz", Piracicaba, Brasil. 56 p.

TAMM, C.O., 1964. Determination of Nutrient Requirements of Forest Stands. International Review of Forestry Research 1: 115-170. 